\title{
Fitness effects and transmission routes of a microsporidian parasite infecting Drosophila and its parasitoids
}

\author{
P. H. FUTERMAN ${ }^{1}$, S. J. LAYEN ${ }^{1}$, M. L. KOTZEN ${ }^{1}$, C. FRANZEN ${ }^{2}$, A. R. KRAAIJEVELD ${ }^{1 *}$ \\ and H. C. J. GODFRAY ${ }^{1}$ \\ ${ }^{1}$ NERC Centre for Population Biology, Division of Biology, Imperial College London, Silwood Park Campus, Ascot, \\ Berkshire SL5 7PY, UK \\ ${ }^{2}$ Klinik und Poliklinik für Innere Medizin I, Universität Regensburg, Franz-Fosef-Strauß Allee 11, D93042 Regensburg, \\ Germany
}

(Received 25 August 2005; revised 7 October 2005; accepted 7 October 2005; first published online 1 December 2005)

\begin{abstract}
SUMMARY
A microsporidian infection was discovered in laboratory cultures of Drosophila species. Ultrastructural examination suggested it belonged to the poorly characterized species Tubulinosema kingi, and morphological and sequence data are presented. We explored how T. kingi affected the fitness of Drosophila melanogaster and D. subobscura, as well as the fitness of 2 of their parasitoids, Asobara tabida and Pachycrepoideus vindemiae. In Drosophila, infections caused changes in most of the traits we looked at that were associated with fitness, in particular causing a 34-55\% reduction in early-life fecundity. Parasitoid fitness was affected more severely by infection than that of their hosts, with pupal mortality in particular increasing by $75-89 \%$. We investigated the most important routes of transmission for T. kingi in a laboratory setting. Letting Drosophila larvae feed on medium contaminated with spores from infected dead flies resulted in $100 \%$ infection. Low levels of transmission $(<10 \%)$ were found between larvae, and vertically between mothers and their offspring. Parasitoids developing in infected hosts all became infected, but infected adults were neither able to transmit the pathogen to their offspring nor to their offspring's Drosophila host, either directly, or via contamination of the ovipositor or other body parts. A field survey of Drosophila and their parasitoids in southern England revealed no natural infections. We discuss the potential importance of Microsporidia in parasitoid-host interactions, and for those working with Drosophila in the laboratory.
\end{abstract}

Key words: Tubulinosema kingi, Drosophila, Asobara tabida, Pachycrepoideus vindemiae, microsporidian parasite, fitness costs, transmission.

\section{INTRODUCTION}

Microsporidia are common intracellular parasites, infecting a wide range of hosts, including humans. They were originally thought to be primitive protozoa because of their lack of mitochondria, but most recent studies suggest they are actually highly derived fungi (Hirt et al. 1999; Williams et al. 2002). In all species the infective extracellular life-stage is a spore which contains a coiled up polar filament characteristic of the group. The contents of the spore (sporoplasm) are injected at germination into a host cell through the extruded polar filament. Inside the host cell, the sporoplasm matures and replicates vegetatively as meronts for one or more generations. Meronts then develop into sporonts during which characteristic electron-dense material is deposited on the outside of the plasma membrane. Sporonts divide to become sporoblasts which then mature

* Corresponding author: NERC Centre for Population Biology, Division of Biology, Imperial College London, Silwood Park Campus, Ascot, Berkshire SL5 7PY, UK. Tel: +44-(0)20 75942544. Fax: + 44-(0)133 873173. E-mail: a.kraayeveld@imperial.ac.uk into spores. Wittner and Weiss (1999) and Franzen (2004) provide extensive accounts of Microsporidia structure and life-cycles.

Drosophila and their parasites and pathogens are a very useful model system for studying hostparasite coevolution at an ecological, immunological and molecular genetic level. The natural enemies that have received most attention are hymenopteran parasitoids, nematodes, entomopathogenic fungi and bacteria (Nappi, 1981; Jaenike, 1995; Kraaijeveld, van Alphen and Godfray, 1998; Hoffmann and Reichhart, 2002; Tzou, De Gregorio and Lemaitre, 2002). Little work has been done on the Microsporidia which infect Drosophila, and the role these parasites play in the interactions of Drosophila with its other natural enemies is unexplored. We are aware of records of 3 species of Microsporidia from Drosophila. Tubulinosema kingi (Kramer) (formerly known as Nosema kingi) was first described from D. willistoni (Burnett and King, 1962; Kramer, 1964), but infects a range of Drosophila species, including D. melanogaster (Armstrong, 1976; Armstrong et al. 1986; Armstrong and Bass, $1989 a, b)$. Octosporea muscaedomesticae Flu infects a wide range of dipteran species, including members of 
the families Drosophilidae, Muscidae, Sarcophagidae and Calliphoridae (Kramer, 1973; RoxströmLindquist, Terenius and Faye, 2004). Finally, the recently described Tubulinosema ratisbonensis Franzen et al. is known from a single laboratory culture of D. melanogaster (Franzen et al. 2005).

Infection of parasitoids by the Microsporidia of their hosts is not uncommon (Brooks, 1993; Rosenheim et al. 1995 ; Becnel and Andreadis, 1999). This normally occurs when a parasitoid larva feeds on an infected host. Infected parasitoids can transmit the Microsporidia vertically to their own offspring and horizontally when they oviposit in uninfected hosts. Uninfected parasitoids may also act as 'dirty needles' and transmit Microsporidia via a contaminated ovipositor from infected to uninfected hosts (Brooks, 1993; Becnel and Andreadis, 1999). We know of no published reports of Microsporidia infecting parasitoids of Drosophila.

Microsporidian infections have a variety of effects on the fitness of their hosts. In insects, individuals infected with Microsporidia typically suffer a combination of reduced survival, adult size, longevity and fecundity (Wilson, 1974; Agnew et al. 1999; Schuld, Madel and Schmuck, 1999; Boohene, Geden and Becnel, 2003). However, the magnitude of these fitness reductions varies from relatively minor to highly damaging (Becnel, Garcia and Johnson, 1995; Schuld et al. 1999). Parasitoids in particular often suffer large fitness reductions after infection with their host's Microsporidia (Geden et al. 1995; Schuld et al. 1999; Boohene et al. 2003; Geden, Ferreira de Almeida and Pires do Prado, 2003) and these effects appear to be stronger than those suffered by the host. However, to our knowledge there has been no systematic comparative study of dose-response patterns in host and parasitoid pairs.

As part of ongoing studies of the co-evolution of host resistance and parasitoid counter-measures we maintain a number of laboratory cultures of Drosophila species and their parasitoids. In 2001 we noticed a relatively high frequency of parasitoids with physogastric (distended and pale) abdomens which suggested a microbial infection. Light microscopy showed characteristic spores indicating a microsporidial infection, and we subsequently found the same spores in Drosophila. The aims of the study reported here were 4-fold. First, we sought to identify the microsporidian and establish whether it was the same species as those previously reported for Drosophila. Second, we wished to find out whether and by how much the infection affected fly and parasitoid fitness. Earlier work (Armstrong et al. 1986; Armstrong and Bass, 1989a,b) found that T. kingi infections affected a number of traits in $D$. melanogaster that are associated with reduced fitness (Table 1, first column) though, surprisingly, infected larvae showed increased survival and
Table 1. The effects of infection with Tubulinosema kingi on various fitness traits of Drosophila melanogaster

(Data from our study and those of Armstrong and colleagues $\left({ }^{1}\right.$ Armstrong et al. $1986 ;{ }^{2}$ Armstrong and Bass, $1989 a ;{ }^{3}$ Armstrong and Bass, $\left.1989 b\right)$. The figures are the percentage increase or decrease in the trait (* indicates that the difference is significant).)

\begin{tabular}{|c|c|c|}
\hline & $\begin{array}{l}\text { Armstrong } \\
\text { and } \\
\text { colleagues }\end{array}$ & Our study \\
\hline Prepupal period & $+3.9 \%{ }^{(2) *}$ & $+3 \cdot 6 \pm 0 \cdot 8 \% *$ \\
\hline Pupal period & $+4.9 \%{ }^{(2) *}$ & $+2 \cdot 6 \pm 1 \cdot 1 \%$ * \\
\hline Larval mortality & $-68 \cdot 0 \%^{(2) *}$ & Not measured \\
\hline Pupal mortality & $+38.0 \%{ }^{(2)} *$ & $+101 \cdot 9 \pm 32 \cdot 4 \%$ \% \\
\hline Body weight $\hat{\sigma}$ & $+18 \cdot 9 \%^{(2)}$ & Not measured \\
\hline Body weight + & $+20 \cdot 0 \%^{(2) *}$ & Not measured \\
\hline Wing length ? & Not measured & $-1.4 \pm 0.6 \% *$ \\
\hline Fecundity & $-66 \cdot 1 \%{ }^{(3) *}$ & $-33 \cdot 7 \pm 3 \cdot 8 \% *$ \\
\hline Offspring production & $-67 \cdot 4 \%{ }^{(3)} *$ & Not measured \\
\hline Longevity $\hat{0}$ & $-51 \cdot 6 \%^{(3) *}$ & Not measured \\
\hline Longevity $q$ & $-48 \cdot 5 \%^{(3) *}$ & Not measured \\
\hline Longevity (both sexes) & $-26 \cdot 1 \%{ }^{(1)} *$ & Not measured \\
\hline
\end{tabular}

infected male and female flies were heavier. We looked at fitness effects in 2 Drosophila species, D. melanogaster and D. subobscura, in a larval parasitoid, Asobara tabida (Hymenoptera, Ichneumonoidea, Braconidae), and in a more limited way in a pupal parasitoid Pachycrepoideus vindemiae (Hymenoptera, Chalcidoidea, Pteromalidae). Third, we investigated routes of transmission. Armstrong et al. (1986) found $11 \%$ vertical transmission of $T$. kingi in D. melanogaster, though much higher levels (up to $81 \%$ ) in D. willistonii. To do this, we explored whether our microsporidian could be transmitted vertically in the host fly or parasitoid, horizontally between fly larvae or from adult to larvae, and whether infection of the parasitoid is a dead end or if fly larvae can be infected from the spores produced in their natural enemy. We also asked whether transmission through contaminated ovipositors or other body parts (the 'dirty needle' route) could occur. Finally, we surveyed local wild populations of Drosophila and their parasitoids to see if they carried the infection. Our longer-term aims are to learn more about microsporidian infections of Drosophila as the genetic resources available for this host enable the interactions to be studied in a way not possible for any other invertebrate host species.

\section{MATERIALS AND METHODS}

\section{Microsporidian identification}

Ultrastructure. For microscopy, infected flies and wasps were dissected and fixed in $0 \cdot 1 \mathrm{M}$ cacodylatebuffered Karnovsky solution $(2 \cdot 5 \%$ glutaraldehyde and $1 \%$ paraformaldehyde; overnight at room 
temperature). Samples were post-fixed in $1 \%$ osmium tetroxide $(2 \mathrm{~h})$ at $\mathrm{pH} 7 \cdot 3$, dehydrated in graded ethanols, and embedded in EmBed-812 epoxy resin (all reagents from Science Services, Munich/ Germany; automated LYNX-tissue processor Leica, Bensheim, Germany). After $48 \mathrm{~h}$ heat polymerization at $60{ }^{\circ} \mathrm{C}$, semi-thin $(0.8 \mu \mathrm{m})$ sections were cut, stained with toluidine blue and basic fuchsin and, after light microscopical selection of representative tissue areas, the Epon block was trimmed for ultrathin sectioning. Ultrathin $(80 \mathrm{~nm})$ sections were cut with a diamond knife on a Reichert Ultracut-S ultramicrotome (Leica) and double contrasted with aqueous $2 \%$ uranyl acetate and lead citrate solutions for $10 \mathrm{~min}$ each. The sections were examined in a LEO912AB electron microscope (Zeiss, Oberkochen, Germany) operating at $80 \mathrm{kV}$, equipped with a bottom-mounted CCD-camera (Proscan, Lagerlechfeld, Germany) capable of recording pictures at $1024 \times 1024$ pixel resolution. Imaging was done with the AnalySISsoftware, Ver. 3.2 (Soft Imaging System, Münster, Germany).

Molecular data. Ribosomal RNA sequences are commonly used for microsporidian taxonomy and phylogeny (Canning et al. 2002; Müller et al. 2000). To sequence the small subunit (SSU) rRNA gene, we first extracted DNA from infected D. melanogaster using a proteinase $\mathrm{K}$ digestion in $2 \%(\mathrm{w} / \mathrm{v})$ CTAB buffer at $60{ }^{\circ} \mathrm{C}$ for $3 \mathrm{~h}$. This was followed by a single chloroform extraction and protein removal using protein precipitation buffer (Promega, Southampton, UK). DNA was then precipitated in isopropanol, washed with $70 \%$ ethanol, air dried, and re-suspended in $\mathrm{dH}_{2} \mathrm{O}$. PCR was carried out on this template using the primers HA3Bf $\left(5^{\prime}-\mathrm{GTT}\right.$ CCA GCA GTA AAC TAT GCC-3') and HG2r (5'-AAG GAG GAG AAG AAA CTA ACT AGG AT-3'; Gatehouse and Malone, 1998).

Subsequently, we extended this sequence into the large subunit (LSU) using DNA that was isolated from infected wasps. The 3 '-end of the SSU rRNA gene, the intergenic spacer, and the $5^{\prime}$-end of the LSU rRNA gene were amplified with the primers 530f (5'-GTG CCA GC(C/A) GCC GCG G-3') located in the SSU rRNA gene and 580r (5'-GGT CCG TGT TTC AAG ACG G-3'; Vossbrinck et al. 1993) located in the LSU rRNA gene.

\section{Microsporidian screening}

To test for the presence of our Microsporidia, we designed a PCR primer pair based on the specific sequence of its SSU rRNA gene (forward primer: GAC CTA TTG AGG ACA ATC AGT AGC; reverse primer: CTT AGT GAG CTA CGA TTA CTA GGA; primers about 600 bp distant from each other). Primers were designed manually against the desired rRNA regions and then BLASTed to ensure there would be no cross-priming to anything other than microsporidian sequences. As a positive control for DNA, we used a primer pair designed against the insect 28S D2 rRNA gene (Belshaw et al. 1998). DNA was extracted from insects using the Qiagen DNEasy kit, following the manufacturer's instructions for insect DNA extraction. For routine scoring of infection frequency, and to enable semi-quantitative assessment of the level of microsporidian infection of individual insects, we used Giemsa staining (Pell and Canning, 1993). The abdomen of an insect was smeared onto a microscope slide and left to air-dry for $5 \mathrm{~min}$. The slide was then fixed in pure methanol for $10 \mathrm{~min}$ and then transferred to a $10 \%$ solution of Gurr's improved R66 Giemsa stain in $\mathrm{pH} 7.2$ phosphate buffer. Slides were left for $45 \mathrm{~min}$ and then rinsed under running water. Excess water was gently blotted from the slides which were then left to dry. We examined the slides under oil immersion at $1000 \times$ magnification and scored the level of microsporidian infection as 'none' (when no spores were seen), 'light' (when just a few scattered spores were observed), 'medium' (when spores were more numerous and more widespread) or 'heavy' (when large masses of spores were observed).

\section{Microsporidian-free insect cultures}

Flies. Microsporidian infections were first detected in laboratory cultures of Drosophila melanogaster and their parasitoids and to study their effects on the host it was first necessary to obtain infection-free cultures. For D. melanogaster we used a strain originally collected in Lyon, France in 1995 and since then maintained in the laboratory as a large, outbred population. Several hundred females were allowed to lay eggs for a few hours. These eggs were surfacesterilized by immersion in $0.6 \% \mathrm{NaOCl}$ solution for $5 \mathrm{~min}$ to guard against the possibility of transovum vertical transmission and then washed several times. About 300 surface-sterilized, de-chlorinated eggs were placed individually in small $(50 \times 10 \mathrm{~mm})$ sterile glass vials containing yeast/sugar medium and live baker's yeast. Upon emergence, 28 females were paired with males and allowed to oviposit in larger $(80 \times 20 \mathrm{~mm})$ vials until $50-100$ eggs had been laid. All the male and female flies were then killed and PCR-screened for Microsporidia. None were parasitized and the infection-free D. melanogaster culture was established from their pooled offspring.

A Microsporidia-free culture of D. subobscura was established in the same way from a strain that had originally been collected in the Netherlands and which had been in laboratory culture for more than 20 years. The infection-free D. subobscura culture was based on the progeny of 27 pairs of flies. 
Parasitoids. To obtain a Microsporidia-free culture of the parasitoid Asobara tabida, we used a strain called 'SOS' that had been collected in France in the 1980s. The parasitoid was maintained on the infection-free D. subobscura culture. About 100 second instar D. subobscura larvae were distributed amongst 6 bottles $(150 \mathrm{ml})$ containing yeast/sugar medium and live baker's yeast. Five female and 3 male parasitoids were placed in each bottle for $16 \mathrm{~h}$, after which the host larvae were removed and washed in $0.6 \% \mathrm{NaOCl}$ solution. Larvae were then reared individually until parasitoids emerged when 24 pairs of male and female $A$. tabida were placed each in a vial with host larvae. After they had parasitized a series of hosts the parasitoids were killed and PCR-screened for microsporidian infection. All proved uninfected and their offspring was pooled to form the Microsporidia-free A. tabida culture.

The Pachycrepoideus vindemiae strain we used was originally collected at Silwood Park, UK, and had been reared in the laboratory on $D$. melanogaster pupae for more than 12 years (Kraaijeveld and Godfray, 2003). Preliminary work had shown that parasitoids which were reared on uninfected hosts were free of infection and therefore we did not set up a separate infection-free culture of this species.

All insect rearing and experiments were done at $20 \pm 1{ }^{\circ} \mathrm{C}$ and 16:8 light: dark regime, apart from work related to $P$. vindemiae, which was conducted at $25 \pm 0 \cdot 5{ }^{\circ} \mathrm{C}$ (the optimal temperature for this species). All work with Microsporidia-free cultures was carried out under sterile conditions with autoclaved equipment and alcohol-swabbed benches and microscopes. Microsporidia-free cultures were regularly screened microscopically to make sure reinfection had not taken place.

\section{Effects of infection on fly and parasitoid fitness}

To study the fitness consequences of infection we compared the performance of insects from our uninfected cultures with individuals from the same culture that had been deliberately exposed to Microsporidia. Nothing is known about natural levels of infection and exposure, and because one of our key aims was to compare relative fitness effects of the microsporidian on flies and parasitoids, we chose an infection method and spore dose which preliminary experiments had shown to lead to clear fitness effects. To infect hosts, microsporidian spores were obtained from heavily-infected parasitoids, the most convenient source of large quantities of spores. Wasps were first surface sterilized using a $1 \% \mathrm{NaOCl}$ and $0 \cdot 1 \% \mathrm{SDS}$ solution to reduce the risk of microbial contamination and then homogenized and filtered in $0 \cdot 1 \%$ SDS. Spore concentrations were measured using a haemocytometer and $\sim 2.5 \times 10^{6}$ spores were pipetted into individual $80 \times 20 \mathrm{~mm}$ vials containing yeast/sugar medium, live baker's yeast and the eggs of the fly species to be infected.

Flies. For D. melanogaster we set up 22 vials each containing 50 eggs, while for the slightly larger D. subobscura we set up 36 vials with half the number of eggs. In both cases, an equal number of control vials received the same volume of $0 \cdot 1 \% \mathrm{SDS}$.

Vials were checked daily and the numbers of pupae and emerging adults recorded to obtain data on development time and mortality. To assess fecundity, we took 2 females and 2 males that had emerged from each of the individual vials and placed them in a series of fresh vials with oviposition medium that were changed daily for a period of 10 days. The numbers of eggs laid per day was recorded. Any males that died were replaced though if females died the replicate was discarded. At the end of the experiment females were killed, measured for size (using a wing venation proxy), and screened for Microsporidia to check the integrity of the treatments. All females in the infected treatment were heavily infected whereas none of the control females showed any sign of infection.

Parasitoids. To study the effects of infection on Asobara tabida fitness 10-15 D. subobscura from the Microsporidia-free culture were placed in each of 50 vials $(80 \times 20 \mathrm{~mm})$ containing oviposition medium and allowed to lay eggs overnight. To 15 vials we added $\sim 2.5 \times 10^{6}$ spores in $0.1 \% \mathrm{SDS}$ and to a further ten $\sim 2.5 \times 10^{4}$ spores, the remaining 25 vials acted as controls and received the same quantity of $0 \cdot 1 \%$ SDS. Four days later 2 female (and 1 male) A. tabida from the microsporidia-free parasitoid culture were placed in each vial and allowed to parasitize the now 2 nd instar larvae for $16 \mathrm{~h}$. Four to five weeks later, the number of successfully emerged parasitoids was counted while unhatched pupae were dissected to obtain a measure of pupal mortality. Effects of infection on parasitoid fecundity were first assessed by dissecting a random sample of 15 adult females from each of the two treatments and the control and counting the number of eggs in both ovaries. We also allowed 10 females from each treatment and control to search for hosts in a Petri dish, lined with agar and containing a yeast patch with 20 uninfected 2 nd instar $D$. subobscura larvae. Parasitoids were removed after $2.5 \mathrm{~h}$ and their infection status confirmed by Giemsa staining as described above. The number of parasitoids emerging from the hosts exposed to attack was counted 4-5 weeks later.

Pachycrepoideus vindemiae is a polyphagous parasitoid that attacks the pupae of many dipteran species. We asked whether its fitness was affected by attacking microsporidian infected hosts. Because the infection-free D. melanogaster strain we used for the earlier experiments was no longer available 
at the time of this experiment, we used a population derived from flies collected in Leiden, the Netherlands, in 1994 as hosts in this experiment. We examined Giemsa-stained smears of 200 individuals of this strain and found none to be infected. We allowed flies to lay eggs in four $150 \mathrm{ml}$ bottles containing oviposition medium. To 2 of the bottles we added $10^{7}$ spores (the surface area of the medium inside the bottle is about 4 times that of a vial, so this spore dose is comparable to what we applied to vials); the other 2 bottles received $0 \cdot 1 \% \mathrm{SDS}$ and served as controls. We allowed the hosts to develop until the pupae were 2-3 days old and then removed them from the bottles, placing 20 infected pupae in each of 10 agar-lined vials with the same number of uninfected pupae in control vials. Two parasitoid females were then added to each vial and allowed to search for hosts until they died. The numbers of flies and parasitoids emerging in each vial were recorded. One week after the last parasitoid emergence, pupae from which nothing had emerged were dissected and whether they contained a dead fly, a dead parasitoid, or were of uncertain fate was recorded. Twenty treatment and control flies were checked for Microsporidia infection by light microscopy, as were 13 and 20 parasitoids that had developed in infected and uninfected hosts respectively (19 parasitoids emerged from infected hosts, but 6 had dried out too much to enable screening, hence the lower number of parasitoids screened from infected hosts).

Fitness traits were analysed with ANOVA, proportion data after angular transformation.

\section{Transmission}

Transmission of the microsporidian infection between flies is potentially vertical or horizontal, and if horizontal the spores may originate from infected larvae or infected adults. Parasitoids almost certainly acquire the infection through feeding on infected hosts, and it is possible that they may transmit the infection to other hosts through a contaminated ovipositor (the 'dirty needle' route) or that adult parasitoids are themselves a source of spores. We carried out a series of experiments to explore and quantify these different routes.

\section{Fly to fly}

For the interpretation of several of the transmission experiments, we needed to know the level of infection in adult flies after exposure of larvae to our standard spore dose. This is determined by the rate at which larvae become infected and the degree to which the infection is retained as the insect matures. To assess this level of infection, we applied $\sim 2.5 \times 10^{6}$ spores to vials with 1-day-old, infection-free $D$. melanogaster larvae (50 larvae per vial, 11 vials) as described above. Upon emergence of the adult flies we screened and assessed the level of infection of 32 flies using Giemsa staining.

To measure larva-to-larva transmission we infected larvae from our standard infection-free strain of D. melanogaster and then allowed them to feed in medium which subsequently was used by larvae from a genetically marked (white-eye) recipient strain. We confirmed the latter was infection-free by both molecular and microscopy screening. Larvae from our infection-free culture were exposed to spores as described above. When they had reached the 3 rd instar, they were washed from the vials and surface-sterilized by immersion in a $1 \% \mathrm{NaOCl}$ and $0 \cdot 1 \%$ SDS solution for several minutes. Fifteen larvae were added to each of 10 vials containing 50 eggs of the white-eyed strain. The vials were stored until the insects completed development whereupon 15 white-eyed flies from each vial were screened by microscopy to assess their level of microsporidian infection.

We assessed adult-to-larva transmission by first exposing uninfected $D$. melanogaster larvae to spores as described previously. Upon emergence, the adult flies were killed by overexposure to $\mathrm{CO}_{2}$. Six cadavers were added to each of 15 vials that contained rearing medium and 3 different densities of fly eggs $(25,50$ and $100 ; 5$ vials each). The flies were allowed to complete development with the cadavers which quickly decomposed, and when they reached the adult stage they were screened for infection by microscopy $(88,100$ and 98 flies were screened in total for the 25,50 and $100 \mathrm{egg}$ treatments respectively).

To measure vertical transmission, uninfected larvae were exposed to spores as described above. Upon emergence, 15 female flies were placed individually in vials with a pair of males and allowed to oviposit for $24 \mathrm{~h}$. The flies were then killed and screened for infection by microscopy, as were a sample of their offspring when they emerged. In total 281 second generation flies were screened.

Fly to parasitoid. To confirm transmission from infected larva to parasitoid we used microscopy to screen for infection 30 adult parasitoids that had developed from the eggs of infection-free adult wasps that had oviposited into infected hosts (as part of the fitness experiment with parasitoids described above).

Parasitoid to parasitoid. We tested whether parasitoids transmitted infections vertically to their offspring by using microscopy to determine the infection status of 151 parasitoids that were the progeny of 30 infected females (produced as part of the experiment to determine the effect of infection on parasitoid fecundity). 


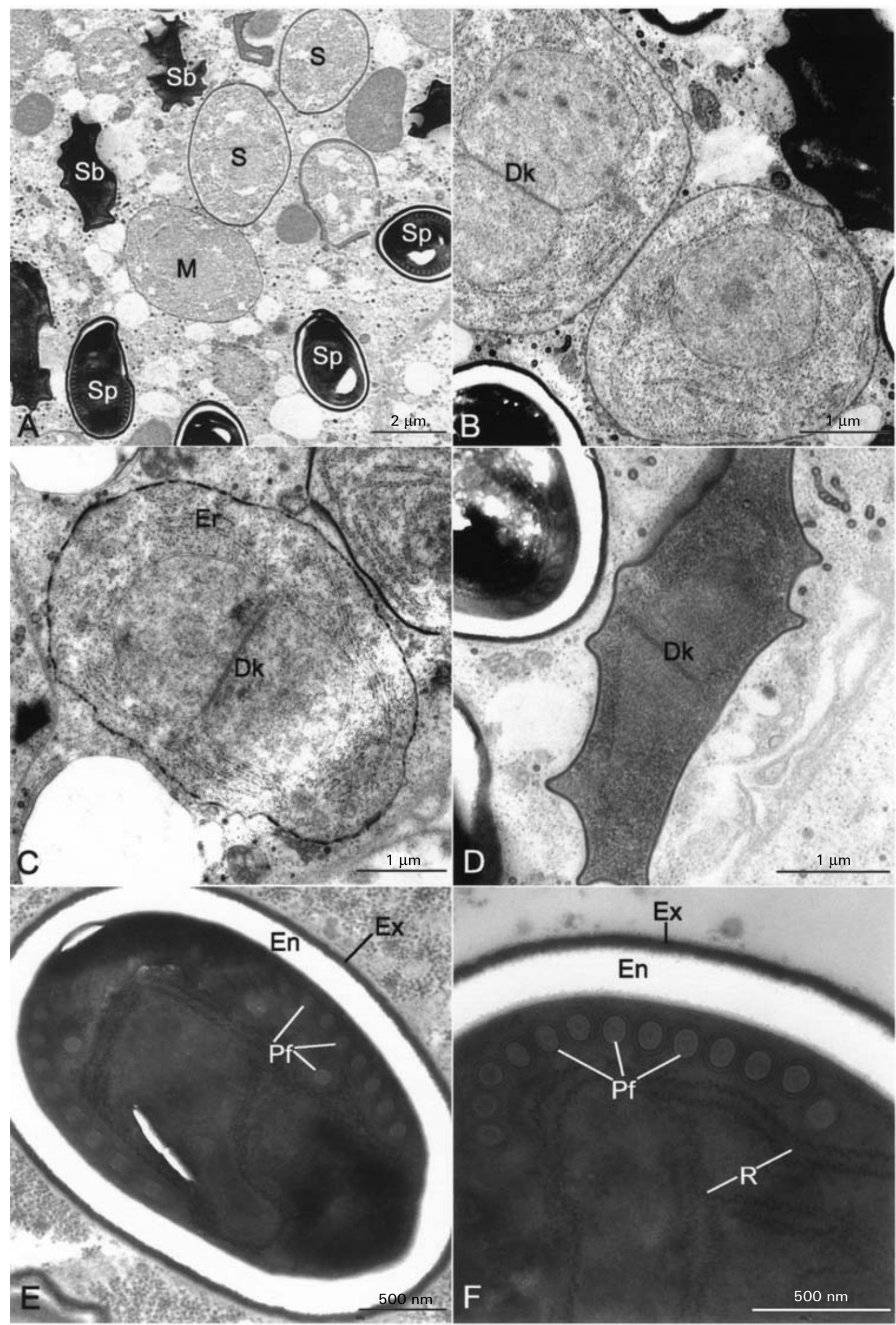

Fig. 1. Transmission electron micrographs of Tubulinosema kingi developmental stages and mature spores. (A) Lowpower micrograph which shows meronts $(\mathrm{M})$, sporonts $(\mathrm{S})$, sporoblasts $(\mathrm{Sb})$, and spores $(\mathrm{Sp})$. (B) Two meronts, 
Parasitoid to fly. After laying eggs in the vertical transmission experiment these same females were used to assess transmission from infected parasitoids to uninfected larvae. The wasps were transferred individually to Petri dishes each containing 20 uninfected D. melanogaster larvae, and allowed to search for hosts for $2 \cdot 5 \mathrm{~h}$. When flies emerged 52 were screened by microscopy for both infection status and for the presence of an encapsulated parasitoid egg. Flies with an encapsulated egg clearly had been parasitized and could have become infected directly from the parasitoid. Flies without a capsule would not have been parasitized though they might have picked up an infection indirectly if the adult parasitoid contaminated the medium with spores.

Parasitoids as vectors. Finally, we assessed whether (uninfected) parasitoids can act as vectors of Microsporidia through contaminated ovipositors. In the experiment to study whether larval parasitoids contracted the infection from infected hosts, Microsporidia-free parasitoids oviposited into infected hosts. These wasps were then transferred to Petri dishes containing uninfected D. melanogaster larvae and allowed to continue searching for hosts for $2.5 \mathrm{~h}$. When flies emerged we dissected 46 individuals and checked by microscopy for both microsporidian infection and an encapsulated parasitoid egg. The presence of both would indicate 'dirty-needle' transmission.

\section{Field prevalence}

During the summer of 2001 we collected wild Drosophila and their parasitoids using insect traps placed in the field at Silwood Park, southern England. The traps were baited with agar mixed with pureed apple and fresh baker's yeast. Insects were collected twice a week from May to August, identified to species and then preserved in $100 \%$ ethanol at $-20{ }^{\circ} \mathrm{C}$. Insects were screened for Microsporidia using the PCR method described above.

\section{RESULTS}

\section{Microsporidian identification}

Morphological and sequencing studies were carried out to establish whether the microsporidian infection could be ascribed to any of the species previously associated with Drosophila. Transmission electron microscopy showed that all life-cycle stages have direct contact with the host cell cytoplasm (Fig. 1A). Meronts were predominantly round or slightly oval in shape and contained a single diplokaryon consisting of 2 nuclei in a coffee bean-like association (Fig. 1B-C). Merogony was by binary fission of elongated cells with 2 pairs of nuclei in diplokaryotic arrangement on both sides. The meront cytoplasm contained numerous free ribosomes but relatively little rough endoplasmic reticulum. On a few meronts the cell membrane had a surface coat of tubular elements that encircled the cell. Sporonts were round or slightly oval, and also contained a diplokaryon. The cell membrane of these cells was covered by electron-dense material. Compared to the cytoplasm of meronts, the cytoplasm of sporonts contained more free ribosomes and considerably more rough endoplasmic reticulum that was often arranged in a regular multilayer pattern (Fig. 1C). Spores were oval or slightly pyriform and diplokaryotic, measuring $4 \cdot 6 \pm 0 \cdot 3 \times 2 \cdot 7 \pm 0 \cdot 1 \mu \mathrm{m}$ (mean \pm s.D., $n=20)$ in fresh smears and $3 \cdot 6 \pm 0 \cdot 3 \times 2 \cdot 4 \pm$ $0 \cdot 2 \mu \mathrm{m} \quad(n=20)$ in fixed smears. Longitudinally sectioned spores exhibited the characteristic organelles of microsporidian spores: exospore, endospore, anchoring disc, polar filament, polaroplast, diplokaryon, ribosomes, and posterior vacuole. The spore wall was composed of an electron-dense exospore without any ornamentation, a thick electron-lucent endospore that was considerably thinner over the anchoring disc, and a plasma membrane (Fig. 1E). The polar filament was slightly anisofilar with the last 3 or 4 coils being smaller in diameter (Fig. 1F). The filament had 10-14 coils that were arranged predominantly in a single row, but in many spores one coil was located inside the outer coils. In some spores the polar filament was irregularly arranged in two or even three rows. Ribosomes arranged in a crystalline pattern as polyribosomes were frequently seen in the spores (Fig. 1E-F). A more detailed description of the ultrastructure of this microsporidium will be given in Franzen et al. (manuscript submitted).

Sequencing of the SSU rRNA gene provided a $1440 \mathrm{bp}$ sequence. The extension into the LSU was $1394 \mathrm{bp}$ long, with a $912 \mathrm{bp}$ region of overlap.

one with a typical diplokaryon (Dk); the cytoplasms contain numerous ribosomes and a few strands of rough endoplasmatic reticulum (Er). (C) Sporont with thickened plasma membrane and a nucleus in diplokaryotic arrangement $(\mathrm{Dk})$. The rough endoplasmatic reticulum $(\mathrm{Er})$ is more frequent and arranged in a regular multilayer pattern. (D) Sporoblast with a typical diplokaryon (Dk) and more condensed cytoplasm as compared to earlier stages. The electron-dense exospore is already present whereas the endospore will be produced later during spore maturation. (E) Spore with an electron-dense exospore (Ex) and a thick electron-lucent endospore (En). The polar filament (Pf) is for the most part arranged in a single row but 2 coils are located inside the outer row. Ribosomes (R) are arranged in a crystalline pattern with polyribosomes visible. (F) Polar filament inside a spore. The polar filament is slightly anisofilar with the last three coils being smaller in diameter and with a different number of layers. 

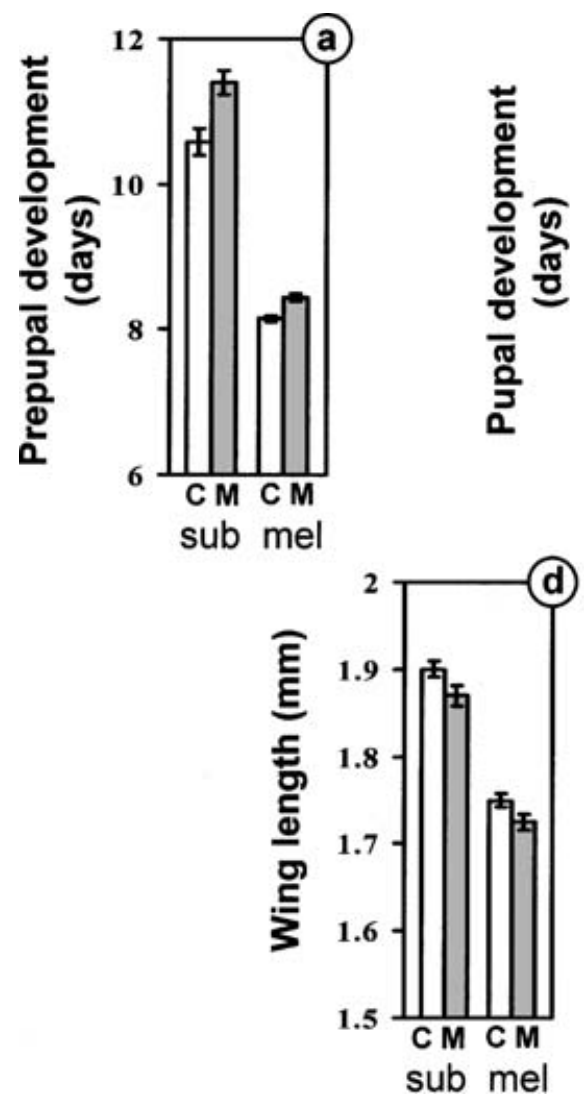
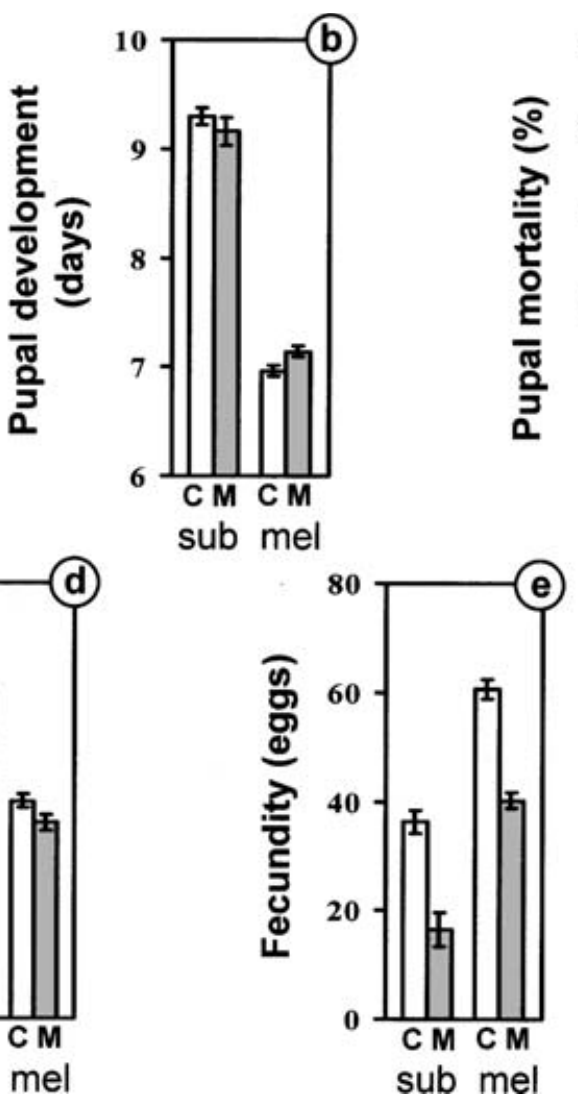

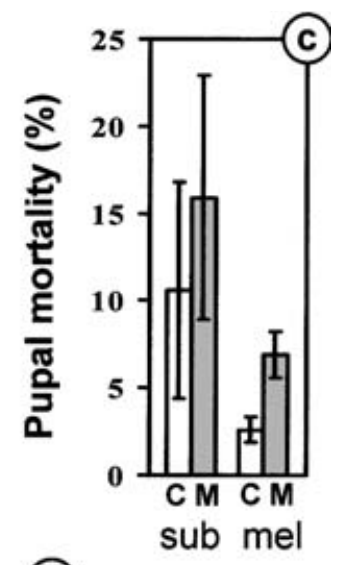

Fig. 2. Effect of Tubulinosema kingi infection on a range of fitness traits in two species of Drosophila (C - white bars: control; M - grey bars: infected; Ds: D. subobscura; Dm: D. melanogaster; error bars: mean \pm s.E.).

We merged the two sequences into one of $1922 \mathrm{bp}$ (GenBank Accession number DQ019419). The merged rRNA gene sequence shows $98 \%$ similarity with the sequence of $T$. ratisbonensis and $98 \%$ similarity with the sequence of $T$. acridophagus (a species described from grasshoppers and formerly known as Nosema acridophagus and 'Visvesvaria' acridophagus; only SSU sequence data are available for this species). The only sequence data available for T. kingi are a $338 \mathrm{bp}$ part of the LSU. Fig. S1 (see Online version of this paper) shows the multiple alignment of the sequence of our species with the GenBank sequences of $T$. acridophagus, T. ratisbonensis and $T$. kingi. If we only consider the partial LSU sequence data, our species is identical to $T$. ratisbonensis and $97 \%$ similar to $T$. kingi on a molecular level.

In the Discussion section we further explain our conclusion that the microsporidian infection in our cultures should be referred to as Tubulinosema kingi.

\section{Fitness effects : flies}

Infection with $T$. kingi had a significantly deleterious effect on most components of the fitness of D. melanogaster and D. subobscura (Fig. 2). Larval insects developed more slowly when infected
(Fig. 2a) with an increase in prepupal development time of $7 \cdot 7 \pm 2 \cdot 4 \%$ in D. subobscura $\left(\mathrm{t}_{32}=3 \cdot 24\right.$, $P=0.0028)$ and $3 \cdot 6 \pm 0.8 \%$ in D. melanogaster $\left(\mathrm{t}_{20}=\right.$ $4.43, P=0.00026)$. The length of the pupal period itself (Fig. 2b) was slightly, but not significantly $(0 \cdot 7 \pm 1.9 \%)$, reduced in D. subobscura $\left(\mathrm{t}_{28}=0.39\right.$, $P=0 \cdot 70)$ and increased by $2 \cdot 6 \pm 1 \cdot 1 \%$ in D. melanogaster $\left(\mathrm{t}_{19}=2 \cdot 42, P=0 \cdot 026\right)$.

Pupal mortality in D. melanogaster was low (Fig. 2c) but increased 101.9 $\pm 32 \cdot 4 \%$ in infected flies $\left(\mathrm{t}_{19}=3 \cdot 15, \quad P=0 \cdot 0053\right)$. Mortality also showed a $46 \cdot 9 \pm 80 \cdot 7 \%$ increase in D. subobscura though this was not significant $\left(\mathrm{t}_{32}=0 \cdot 58, P=0.565\right)$. However, pupal mortality in this species was, for unknown reasons, very variable and we estimate that the smallest effect we could detect (with probability of $0 \cdot 8$ ) was a $255 \%$ increase.

Larval infection led to smaller flies (Fig. 2d), although the reduction in D. subobscura $(1 \cdot 6 \pm 0 \cdot 8 \%)$ was not significant $\left(\mathrm{t}_{30}=1 \cdot 91, P=0 \cdot 066\right)$. D. melanogaster suffered a just-significant reduction in wing length $\left(1 \cdot 4 \pm 0 \cdot 6 \% ; t_{39}=2 \cdot 02, P=0 \cdot 0498\right)$. Infected flies had markedly reduced fecundity (Fig. 2e) with a reduction of $54 \cdot 8 \pm 10 \cdot 7 \%$ in $D$. subobscura $\left(\mathrm{t}_{30}=\right.$ $4 \cdot 78, P<0 \cdot 0001)$ and $33 \cdot 7 \pm 3 \cdot 8 \%$ in D. melanogaster $\left(\mathrm{t}_{39}=8 \cdot 86, P<0 \cdot 000001\right)$.

The fitness effects of $T$. kingi on $D$. melanogaster are summarized in the right column of Table 1 , to 

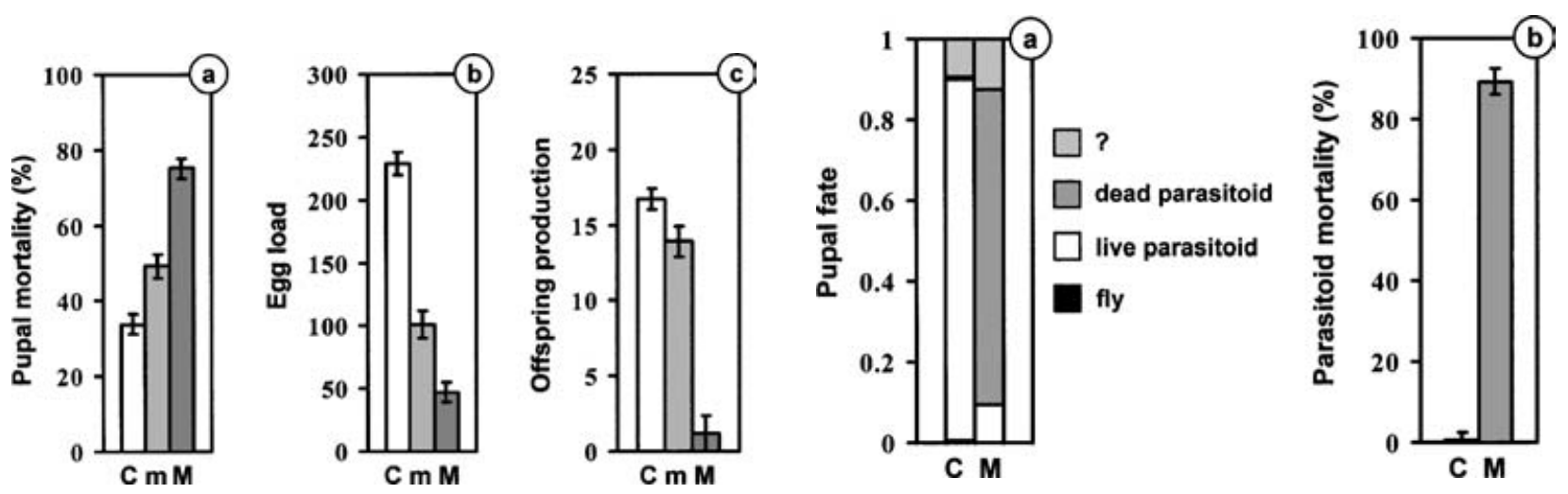

Fig. 3. Effect of Tubulinosema kingi infection on several fitness traits in Asobara tabida $(\mathrm{C}$ - white bars : control; $\mathrm{m}$ - light grey bars : infected with low microsporidian dose; $\mathrm{M}-$ dark grey bars : infected with high microsporidian dose; error bars: mean \pm s.E.).

allow direct comparison with the data obtained by Armstrong et al. (1986) and Armstrong and Bass $(1989 a, b)$.

\section{Fitness effects : parasitoids}

Infection with $T$. kingi detrimentally affected the 3 components of Asobara tabida fitness we measured, predominantly in a dosage-dependent manner (Fig. 3). Infected flies experienced significantly higher rates of pupal mortality $\left(\mathrm{F}_{2,25}=10 \cdot 07, P=\right.$ 0.0006 ; Fig. 3a) with increases of $45 \cdot 6 \pm 27 \cdot 1 \%$ after a low spore dose and $122 \cdot 2 \pm 28 \cdot 2 \%$ after a high dose. Egg load was reduced by $55 \cdot 7 \pm 6 \cdot 1 \%$ in the low-dose treatment and $79 \cdot 4 \pm 5 \cdot 3 \%$ in the high-dose treatment $\left(\mathrm{F}_{2,42}=99 \cdot 50, P<10^{-6}\right.$; Fig. 3b). Finally, offspring production decreased by $16 \cdot 8 \pm 6 \cdot 9 \%$ in the low-dose treatment and $92 \cdot 8 \pm 8 \cdot 3 \%$ in the high-dose treatment $\left(\mathrm{F}_{2,27}=73 \cdot 51, P<10^{-6}\right.$; Fig. $\left.3 \mathrm{c}\right)$.

The vast majority of pupae parasitized by $P$. vindemiae developed into a parasitoid (dead or alive), with only $\sim 10 \%$ being unclassifiable and just a single individual producing a fly (Fig. 4a). The infection status of the pupae had a major effect on whether the parasitoid successfully emerged: in uninfected pupae only 1 of 180 individuals $(0 \cdot 6 \%)$ died while in infected pupae 155 of $174(89 \cdot 1 \%)$ succumbed $\left(\mathrm{t}_{18}=19 \cdot 82, P \ll 10^{-6}\right.$; Fig. $\left.4 \mathrm{~b}\right)$. None of the parasitoids emerging from uninfected pupae were infected by $T$. kingi, whereas the 13 parasitoids we screened from infected pupae were all heavily infected.

\section{Transmission}

The results of all the transmission experiments are summarized in Fig. 5. We first ask whether T. kingi infection is ever lost during metamorphosis. All adult flies from the treatment in which larvae were exposed to high doses of spores carried T. kingi infections,

Fig. 4. (a) Fate of Drosophila melanogaster pupae (C: control; M: Tubulinosema kingi-infected) exposed to Pachycrepoideus vindemiae. Black: fly surviving; white: emerging parasitoid; grey: dead parasitoid: stippled: unrecognizable mass. (b) Mortality of parasitoids among those pupae in which a parasitoid developed $(\mathrm{C}-$ white bar: control; M-grey bar: T. kingi-infected; error bars: mean \pm s.e.).

and the levels of infection were all assessed as heavy. The data are consistent with infections never being lost under these conditions, and if we assume that loss is an independent binomial event then our sample size indicates a lower $95 \%$ confidence limit of $89 \cdot 1 \%$.

In the experiment in which adult infected flies were allowed to die and contaminate the larval medium, we again found $100 \%$ transmission, independent of larval density. Moreover, all larval infections were also assessed as heavy. This suggests that consumption of contaminated food is an important transmission route. Assuming once more binomial infection, our sample size allows us to place a lower $95 \%$ confidence limit for transmission under these conditions of $98 \cdot 7 \%$.

We found some evidence for larva-to-larva transmission though at a much lower rate than from adult to larva. Only $2 \cdot 5 \pm 1 \cdot 5 \%$ of uninfected individuals reared in the presence of infected larvae developed into adult flies carrying Microsporidia. Vertical transmission occurred infrequently, with $9 \cdot 7 \pm 1 \cdot 3 \%$ of the offspring of infected female flies also being infected. In both these cases, the level of infection in all individuals was assessed as light.

We found $100 \%$ transmission from infected host larvae to the parasitoids that develop inside them, with all emerging parasitoids being assessed as having heavy levels of infection. The lower confidence limit for this transmission route is $88.4 \%$. We found no vertical transmission of T. kingi from infected parasitoids to their offspring in our experiments (binomially derived upper confidence limit, $2 \cdot 4 \%$ ). No infection was found in flies which, as larvae, had either been parasitized by infected parasitoids or had been in an otherwise uncontaminated patch visited by infected parasitoids (binomially derived upper confidence limit, 6.8\%). 


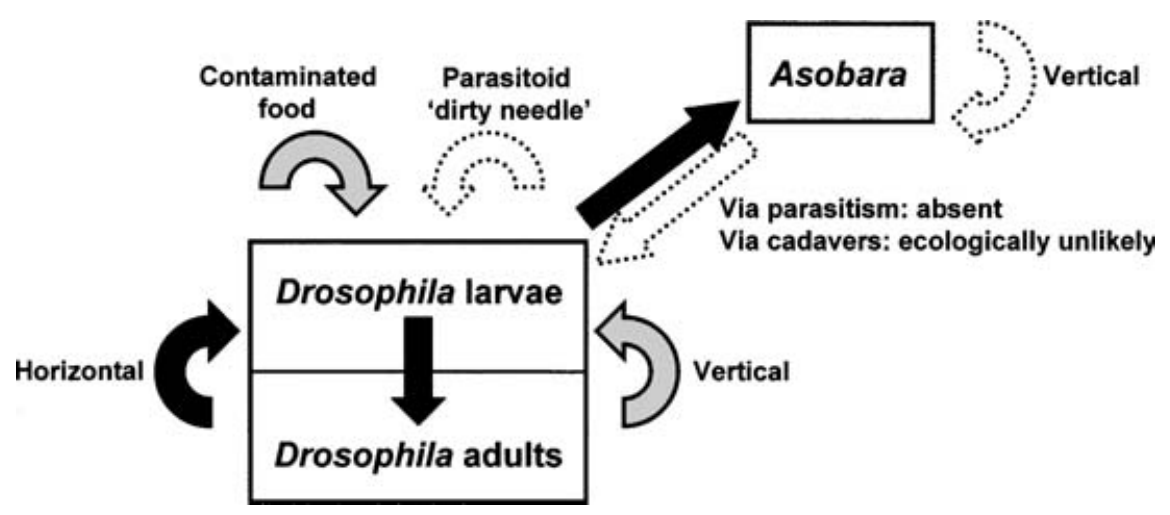

Fig. 5. Summary of the results of the transmission experiments. A white dashed arrow indicates no transmission, a grey arrow $<10 \%$ transmission and a black arrow $100 \%$ transmission.

Finally, we found no evidence that uninfected parasitoids could transmit spores via their ovipositor or other parts of their body (binomially derived upper confidence limit, $7 \cdot 7 \%$ ). It should be noted, however, that due to the low level or resistance of the infection-free fly strain against $A$. tabida, only 16 flies in the last 2 experiments contained an encapsulated parasitoid egg. Therefore, our power to detect transmission as a result of direct parasitism (either by infected or uninfected parasitoids) is limited in these experiments (binomially derived upper confidence limit, 20.6\%).

\section{Field prevalence}

During the 4 months the traps were out in the field, we collected a total of 227 insects (196 flies belonging to 10 species; 31 parasitoids belonging to 2 species). The majority of flies belonged to 4 species (number of individuals caught in parentheses): D. obscura (101), D. subobscura (53), D. phalerate (25) and D. immigrans (17) while the parasitoids were A. tabida (27) and Leptopilina heterotoma (4). Only 1 fly (1 of 4 D. melanogaster we caught) and none of the parasitoids was found to be infected with T. kingi.

\section{DISCUSSION}

\section{Species identification}

The ultrastructure and biology of the Microsporidia infecting our flies and parasitoids place them clearly in the family Tubulinosematidae and the genus Tubulinosema (Franzen et al. 2005). They are diplokaryotic and develop in direct contact with the host cell cytoplasm. Merogonial division is by binary fission of cells with 4 nuclei in diplokaryotic arrangement. No plasmodial stages were observed and sporogony is disporoblastic. The spores are slightly pyriform with a thick endospore that is thinner over the anchoring disc. The polar filament inside the spores is slightly anisofilar and the distal coils have a smaller diameter. Some meronts have small tubuli on the surface. However our species differs from $T$. ratisbonensis, which has been recorded in Drosophila, as well as from the relatively wellstudied T. acridophagus which attacks Orthoptera. Compared to these species, the tubular elements on the meronts seem to be a very transitory and only visible infrequently (Street and Henry, 1993; Franzen et al. 2005). In $T$. ratisbonensis and T. acridophagus, the polar filament is always arranged in a single row along both sides of the diplokaryon (Street and Henry, 1993; Franzen et al. 2005). In spores of our species it is often arranged in a single row, but in many spores 1 coil is located inside the outer coils, and in some spores the polar filament is irregularly arranged in 2 or even 3 rows. A similar arrangement of the polar tube was described by Burnett and King (1962) for T. kingi and illustrated in their line drawing of a spore. Unfortunately, no other ultrastructural data for $T$. kingi have ever been published and the original material is now lost.

We had just under $2000 \mathrm{bp}$ sequence data from the LSU and the SSU of our species and that of $T$. ratisbonensis and T. acridophagus. The sequence similarity to both was relatively high (98\%) though not identical. The only sequence data in GenBank for T. kingi is a short $338 \mathrm{bp}$ section of the LSU deposited by Baker et al. (1994), and to this both our species and T. ratisbonensis showed $97 \%$ similarity. However, the microsporidian from which this sequence was obtained was never studied ultrastructurally, and its identification with T. kingi, sensu Burnett and King (1962), is open to question.

The 2 Microsporidia described from Drosophila differ in minor but consistent ultrastructural features, and our species matches best $T$. kingi. The sequence data show that our species was as distinct from $T$. ratisbonensis as it was from $T$. acridophagus which attacks very different hosts. These two lines of evidence lead us to assign provisionally our Microsporidia to $T$. kingi pending further work on the genus Tubulinosema. In doing this we have decided to discount the GenBank sequence currently associated with $T$. kingi as unconfirmed. 
Tubulinosema kingi biology

Our study extends the host range of $T$. kingi from $D$. melanogaster and D. willistoni to a further species of the same genus, D. subobscura, and 2 of their hymenopteran parasitoids (the ichneumonoid A. tabida and the chalcidoid P. vindemiae).

Infection of Drosophila by T. kingi causes changes in almost all the traits we surveyed that we believe are associated with fitness. This is in general agreement with the results obtained by Armstrong and Bass $(1989 a, b)$, although it should be noted that host genotype, spore dose and temperature all differ in the two sets of experiments. Fecundity was most strongly affected both in our experiments and those of Armstrong and Bass (1989b). In addition, Armstrong et al. (1986) and Armstrong and Bass (1989b) also reported a strong reduction (26-52\%) in longevity, a trait we did not measure. Finally, we found little indication that D. melanogaster and $D$. subobscura are differentially affected by $T$. kingi infection.

D. melanogaster has a well-developed immune system to combat microbial pathogens such as bacteria and fungi (Hoffmann and Reichhart, 2002; Tzou, De Gregorio and Lemaitre, 2002). We know of no studies of defence against Microsporidia in this species, and found no evidence of a successful defensive response against Microsporidia in either of the Drosophila we studied. At least against macroparasites such as parasitoids, D. melanogaster has a much stronger immune response than $D$. subobscura (Kraaijeveld and van Alphen, 1993; Kraaijeveld and van der Wel, 1994) but this was not reflected in any difference in susceptibility to T. kingi.

Compared to their hosts, infected A. tabida parasitoids appear to suffer a much more severe reduction in fitness: pupal mortality more than doubles, the egg load of those parasitoids that manage to survive is reduced by more than three quarters, and actual offspring production to less than a tenth. Even a 100 -fold dilution of the microsporidian dose fed to the fly larvae still causes a substantial fitness reduction in the emerging parasitoids. The severe fitness effect of the microsporidia on parasitoids does not appear to be species-specific. P. vindemiae, a species belonging to a different superfamily, suffers an even higher level of mortality after infection. There are several possible explanations for the greater fitness effects suffered by parasitoids, which are not mutually exclusive. First, parasitoids may experience a higher effective spore dose than their hosts because the pathogen can be present at very high densities in host tissues. Second, parasitoid development time is approximately double that of their hosts and this may allow more time for Microsporidia densities within the insect to build up. Third, Microsporidia may be selected to moderate their virulence in flies if they, and not parasitoids, act as vectors of the pathogen from one Drosophila breeding site to another (see also below).

Vertical transmission of Microsporidia is common (Becnel and Andreadis, 1999; Wittner and Weiss, 1999; Dunn, Terry and Smith, 2001; Terry et al. 2004), but we found only limited evidence for it in our system. D. melanogaster transmitted the infection to their offspring $9 \cdot 7 \%$ of the time which is very similar to the $11 \%$ reported by Armstrong et al. (1986). Vertical transmission of Microsporidia may take place within or, less commonly, on the surface of the host's egg (Undeen, 1997; Wittner and Weiss, 1999). Our success in clearing infections in populations of D. melanogaster and D. subobscura by surface sterilization suggests that these microsporidia are transmitted on the outside of the egg. In contrast, Armstrong (1976) found high levels of transovarial transmission of $T$. kingi in D. willistoni. Armstrong (1977) also demonstrated very high levels (up to $93 \%$ ) of venereal transmission of T. kingi in D. willistoni: infected males transferred the microsporidia to uninfected females and subsequently to their offspring. We found no evidence of vertical transmission from adult $A$. tabida to either their progeny or to the host fly they were attacking.

The major route through which the infection seems to be transmitted is from adult flies dying on and contaminating larval medium, though there was some evidence for low rates of transmission from larva-to-larva. In the field D. melanogaster and related species with similar biology are found in relatively ephemeral habitats such as fruit and sap fluxes which typically are in existence for a single or, in the case of sap fluxes, just a few fly generations. An efficient means of colonizing new habitats is thus likely to be essential for the persistence of the infection, and hence transmission through the adult is likely to be advantageous to the microsporidian. We did not explicitly test whether adult infected parasitoids dying on the larval medium could lead to transmission though think this is likely considering the number of spores they contain. However, infected parasitoids are so disabled and barely capable of flight that this is unlikely to be significant in the field.

We know of no information on the prevalence and infection levels of Tubulinosema in field populations of Drosophila and their parasitoids; previous studies have all involved infections discovered in laboratory populations of flies. This means that the relative importance of the various routes of transmission that we found under laboratory conditions, using heavily-infected individuals, may not necessary apply to the field (although there is also the possibility that this is purely an infection of laboratory flies). Our survey of a Drosophila-parasitoid community in southern England during the summer of 2001 revealed a single infected D. melanogaster, 
but as the survey was done on a university campus we suspect this may have been a laboratory escape. It would be very interesting to survey further wild populations of Drosophila, especially those from warmer climates where D. melanogaster is more common and less associated with man.

Recently, Clark et al. (2005) warned about the widespread prevalence of Wolbachia in Drosophila cultures and the effect this may have on the outcome of fitness experiments. How widespread T. kingi is in laboratory cultures of Drosophila is unknown, but the ease with which flies become infected, and its relatively benign fitness effects, suggest the parasite may be present and undetected in many cultures worldwide. This may have implications for the outcome of experiments, especially if the level of infection varies. The bleaching method we used to set up infection-free cultures shows that flies can be cured from the parasite relatively easily, while monitoring is straightforward both microscopically and molecularly. Maintaining the cultures in such a way to avoid adult cadavers from contaminating the medium will help to keep cultures free of infection. Microsporidian levels may especially build up in cultures maintained in long-term population cages as opposed to those cultured in fresh bottles each generation. Circumstantial evidence for this comes from (unpublished) experiments in which we let $P$. vindemiae parasitize pupae from $6 \mathrm{D}$. melanogaster strains in our laboratory, 4 of which were maintained in bottles, while the remaining 2 were kept in long-term population cages. Pupal mortality of the parasitoid was around $75 \%$, we believe from microsporidian infection, in the 2 strains kept in population cages compared to less than $1.5 \%$ for the 4 strains cultured in bottles.

In the ecological literature there is much current interest in the community and population dynamical effects of intraguild predation (IGP) which occurs when a natural enemy attacks not only a lower trophic level but members of its own trophic level as well (Polis, Myers and Holt, 1989; Rosenheim et al. 1995; Müller and Brodeur, 2002). IGP is also of applied importance as interactions between different natural enemies can lessen their controlling effect on a pest species. Though, as the name suggests, most attention has concentrated on predators, any natural enemy can potentially cause these effects, including $T$. kingi because it attacks both host and parasitoid. Indeed, because it reduces parasitoid fitness more than that of the host, IGP effects in this interaction are likely to be particularly strong. The picture is further complicated by the interaction of $T$. kingi with the pupal parasitoid $P$. vindemiae. The latter is itself an intraguild predator because it is a facultative hyperparasitoid and does not distinguish between pupae containing healthy flies or developing larval parasitoids (van Alphen and Thunnissen, 1983). Models suggest that the effect of intraguild predation on the dynamics of the host is especially unpredictable when pathogens or facultative hyperparasitoids are involved (Rosenheim et al. 1995 and references therein) and this system might be very useful in testing a variety of different aspects of IGP theory.

We thank Rebecca Terry and Alison Dunn (University of Leeds) for sequencing the small subunit rRNA, Josef Schroeder for help with the transmission electron microscopy studies, Rob Butcher for assistance with the development of the molecular screen, Maria Giannakou (University College London) who provided the white-eyed Drosophila strain, Roshan Vijendravarma for assistance with the Pachycrepoideus experiment, and 3 anonymous referees for comments on previous versions of the manuscript. Finally, we are particularly grateful to Liz Canning for much help and advice on Microsporidia.

\section{REFERENCES}

\section{Agnew, P., Bedhomme, S., Haussy, C. and}

Michalakis, Y. (1999). Age and size at maturity of the mosquito Culex pipiens infected by the microsporidian parasite Vavraia culicis. Proceedings of the Royal Society of London, B 266, 947-952.

Van Alphen, J. J. M. and Thunnissen, I. (1983). Host selection and sex allocation by Pachycrepoideus vindemiae Rondani (Pteromalidae) as a facultative hyperparasitoid of Asobara tabida Nees (Braconidae; Alysiinae) and Leptopilina heterotoma (Thomson)(Cynipoidea; Eucoilidae). Netherlands Fournal of Zoology 33, 497-514.

Armstrong, E. (1976). Transmission and infectivity studies on Nosema kingi in Drosophila willistoni and other Drosophilids. Zeitschrift für Parasitenkunde 50, 161-165.

Armstrong, E. (1977). Transmission of Nosema kingi to offspring of Drosophila willistoni during copulation. Zeitschrift für Parasitenkunde 53, 311-315.

Armstrong, E. and Bass, L. (1989a). Effects of Nosema kingi on the development and weight of adult Drosophila melanogaster (OR-R-Strain). Fournal of Invertebrate Pathology 53, 102-106.

Armstrong, E. and Bass, L. (1989b). Nosema kingi: Effects on fecundity, fertility and longevity of Drosophila melanogaster. Fournal of Experimental Zoology 250, $82-86$.

Armstrong, E., Bass, L., Staker, K. and Harrell, L. (1986). A comparison of the biology of a Nosema in Drosophila melanogaster to Nosema kingi in Drosophila willistoni. Fournal of Invertebrate Pathology 48, 124-126.

Baker, M. D., Vossbrinck, C. R., Maddox, J. V. and Undeen, A. H. (1994). Phylogenetic relationships among Vairimorpha and Nosema species (Microspora) based on ribosomal RNA sequence data. Fournal of Invertebrate Pathology 64, 100-106.

Becnel, J. J. and Andreadis, T. G. (1999). Microsporidia in insects. In The Microsporidia and Microsporidiosis (ed. Wittner, M. and Weiss, L. M.), pp. 447-501. ASM Press, Washington DC.

Becnel, J. J., Garcia, J. J. and Johnson, M. A. (1995). Edhazardia aedis (Microspora: Culicosporidae) effects 
on the reproductive capacity of Aedes aegypti (Diptera: Culicidae). Fournal of Medical Entomology 32, 549-553.

Belshaw, R., Fitton, M., Herniou, E., Gimeno, C. and Quicke, D. L. J. (1998). A phylogenetic reconstruction of the Ichneumonoidea (Hymenoptera) based on the D2 variable region of $28 \mathrm{~S}$ ribosomal RNA. Systematic Entomology 23, 109-123.

Boheene, C. K., Geden, C. J. and Becnel, J. J. (2003). Development of microsporidia-infected Muscidifurax raptor (Hymenoptera: Pteromalidae) at different temperatures. Biological Control 26, 1-7.

Brooks, W. M. (1993). Host-parasitoid-pathogen interactions. In Parasites and Pathogens of Insects: Pathogens (ed. Beckage, N. E., Thompson, S. N. and Federici, B. A.), pp. 231-272. Academic Press, San Diego.

Burnett, R. G. and King, R. C. (1962). Observations on a microsporidian parasite of Drosophila willistoni Sturtevant. Fournal of Insect Pathology 4, 104-112.

Canning, E. U., Refardt, D., Vossbrinck, C. R., Okamura, B. and Curry, A. (2002). New diplokaryotic microsporidia (Phylum Microsporidia) from freshwater bryozoans (Bryozoa, Phylactolaemata). European Fournal of Protistology 38, 247-265.

Clark, M. E., Anderson, C., Cande, J. and Karr, T. (2005). Widespread prevalence of Wolbachia in laboratory stocks and implications for Drosophila research. Genetics 170, 1667-1675.

Dunn, A. M., Terry, R. S. and Smith, J. E. (2001). Transovarial transmission in the microsporidia. Advances in Parasitology 48, 57-100.

Franzen, C. (2004). Microsporidia: how can they invade other cells? Trends in Parasitology 20, 275-279.

Franzen, C., Fischer, S., Schroeder, J., Schölmerich, J. and Schneuwly, S. (2005). Morphological and molecular investigations of Tubulinosema ratisbonensis gen. nov., sp. nov. (Microsporidia: Tubulinosematidae fam. nov.), a parasite infecting a laboratory colony of Drosophila melanogaster (Diptera: Drosophilidae). Fournal of Eukaryotic Microbiology 52, 141-152.

Gatehouse, H. S. and Malone, L. A. (1998). The ribosomal RNA gene region of Nosema apis (Microspora): DNA sequence for small and large subunit rRNA genes and evidence for a tandem repeat unit size. Fournal of Invertebrate Pathology 71, 97-105.

Geden, C. J., Ferreira De Almeida, M. A. and Pires do Prado, A. (2003). Effects of Nosema disease on fitness of the parasitoid Tachinaephagus zealandicus (Hymenoptera: Encyrtidae). Environmental Entomology 32, 1139-1145.

Geden, C. J., Long, S. J., Rutz, D. A. and Becnel, J. J. (1995). Nosema disease of the parasitoid Muscidifurax raptor (Hymenoptera: Pteromalidae): prevalence, patterns of transmission, management, and impact. Biological Control 5, 607-614.

Hirt, R. P., Logsdon Jr, J. M., Healy, B., Dorey, M. W., Doolittle, W. F. and Embley, T. M. (1999). Microsporidia are related to fungi: evidence from the largest subunit of RNA polymerase II and other proteins. Proceedings of the National Academy of Sciences, USA 96, 580-585.
Hoffmann, J. A. and Reichhart, J.-M. (2002).

Drosophila innate immunity: an evolutionary perspective. Nature Immunology 3, 121-126.

Jaenike, J. (1995). Interactions between mycophagous Drosophila and their nematode parasites: from physiological to community ecology. Oikos 72, 235-244.

Kraaijeveld, A. R. and van Alphen, J. J. M. (1993). Successful invasion of North America by two Palearctic Drosophila species (Diptera: Drosophilidae): a matter of immunity to local parasitoids? Netherlands Fournal of Zoology 43, 235-241.

Kraaijeveld, A. R., van Alphen, J. J. M. and Godfray, H. C. J. (1998). The coevolution of host resistance and parasitoid virulence. Parasitology 116, S29-S45.

Kraaijeveld, A. R. and Godfray, H. C. J. (2003). Potential life history costs of parasitoid avoidance in Drosophila melanogaster. Evolutionary Ecology Research 5, 1251-1261.

Kraaijeveld, A. R. and Van Der Wel, N. N. (1994). Geographic variation in reproductive success of the parasitoid Asobara tabida in larvae of several Drosophila species. Ecological Entomology 19, 221-229.

Kramer, J. P. (1964). Nosema kingi sp. n., a microsporidian from Drosophila willistoni Sturtevant, and its infectivity for other muscoids. Fournal of Insect Pathology 6, 491-499.

Kramer, J. P. (1973). Susceptibility of sixteen species of muscoid flies to the microsporidean parasite Octosporea muscaedomesticae. Fournal of the New York Entomological Society 81, 50-53.

Müller, A., Trammer, T., Chioralia, G., Seitz, H. M., Diehl, V. and Franzen, C. (2000). Ribosomal RNA of Nosema algerae and phylogenetic relationship to other microsporidia. Parasitology Research 86, 18-23.

Müller, C. B. and Brodeur, J. (2002). Intraguild predation in biological control and conservation biology. Biological Control 25, 216-223.

Nappi, A. J. (1981). Cellular immune response of Drosophila melanogaster against Asobara tabida. Parasitology 83, 319-324.

Pell, J. and Canning, E. (1993). Light microscope and ultrastructural observations of a microsporidian parasite of Mesocyclops rarus (Copepoda: Cyclopoda) in Tanzania. Fournal of Invertebrate Pathology 61, 275-280.

Polis, G. A., Myers, C. A. and Holt, R. D. (1989). The ecology and evolution of intraguild predation: potential competitors that eat each other. Annual Review of Ecology and Systematics 20, 297-330.

Rosenheim, J. A., Kaya, H. K., Ehler, L. E., Marois, J. J. and Jaffee, B. A. (1995). Intraguild predation among biological-control agents: theory and evidence. Biological Control 5, 303-335.

Roxström-Lindquist, K., Terenius, $\mathbf{O}$. and Faye, I. (2004). Parasite-specific immune response in adult Drosophila melanogaster: a genomic study. EMBO Reports 5, 207-212.

Schuld, M., Madel, G. and Schmuck, R. (1999). Impact of Vairimorpha sp. (Microsporidia: Burnellidae) on Trichogramma chilonis (Hymenoptera,

Trichogrammatidae), a hymenopteran parasitoid of the cabbage moth, Plutella xylostella (Lepidoptera, Yponomeutidae). Fournal of Invertebrate Pathology 74, 120-126. 
Street, D. A. and Henry, J. E. (1993). Ultrastructural study of Nosema acridophagus Henry (Microspora: Nosematidae) from grasshopper. Parasitology Research 79, 173-177.

Terry, R. S., Smith, J. E., Sharpe, R. G., Rigaud, T., Littlewood, D. T. J., Ironside, J. E., Rollinson, D., Bouchin, D., Macneil, C., Dick, J. T. A. and Dunn, A. M. (2004). Widespread vertical transmission and associated host sex-ratio distortion within the eukaryotic phylum Microspora. Proceedings of the Royal Society of London, B 271, 1783-1789.

Tzou, P., De Gregorio, E. and Lemaitre, B. (2002). How Drosophila combats microbial infection : a model to study innate immunity and host-pathogen interactions. Current Opinion in Microbiology 5, 102-110.

Undeen, A. (1997). Microsporidia (Protozoa) : A Handbook of Biology and Research Techniques. http:// pearl.agcomm.okstate.edu/scsb387/content.htm
Vossbrinck, C. R., Baker, M. D., Didier, E. S., Debrunner-Vossbrinck, B. A. and Shadduck, J. A. (1993). Ribosomal DNA sequences of Encephalitozoon hellem and Encephalitozoon cuniculi: species identification and phylogenetic construction. Fournal of Eukaryotic Microbiology 40, 354-362.

Williams, B. A. P., Hirt, R. P., Lucocq, J. M. and Embley, T. M. (2002). A mitochondrial remnant in the microsporidian Trachipleistophora hominis. Nature, London 418, 865-869.

Wilson, G. G. (1974). Effects of larval age at innoculation, and dosage of microsporidian (Nosema fumiferanae) spores, on mortality of spruce budworm (Choristoneura fumiferana). Canadian Fournal of Zoology 52, 993-996.

Wittner, M. and Weiss, L. M. (1999). The Microsporidia and Microsporidiosis. ASM Press, Washington DC. 